\title{
Effects of Visual Realism and Moving Detail on Cybersickness
}

\author{
Matti Pouke* \\ University of Oulu
}

\author{
Arttu Tiiro* \\ University of Oulu
}

\author{
Steven M. LaValle ${ }^{\dagger}$ \\ University of Illinois at Urbana-Champaign
}

\author{
Timo Ojala* \\ University of Oulu
}

\begin{abstract}
In this study we compare two conditions of visual detail - modern graphics and detail reduction through cel-shading - in experiencing Cybersickness during virtual movement along a preprogrammed path within a scene depicting a real-world outdoor museum viewed with Oculus CV1. The Cybersickness experience was quantified with the Fast-Motion-Sickness (FMS) scale and the Simulator Sickness Questionnaire (SSQ). We found weak evidence for realistic graphics being more sickness-inducing. Also, FMS scores peaked whenever a participant was entering a building.
\end{abstract}

Index Terms: I.3 [Computer Graphics]: Three-Dimensional Graphics and Realism-Virtual Reality

\section{INTRODUCTION}

Cybersickness, the experience of nausea, disorientation and eyestrain during Virtual Reality (VR) has been one of the greatest problems of VR as well as military simulators throughout their existence $[10,11]$. Depending on the source, Cybersickness is also called Virtual Reality sickness, Visually Induced Motion Sickness (VIMS), or Simulator Sickness (albeit it has been stated that Simulator Sickness symptoms are not exactly the same as in Cybersickness [11]). Many immersive aspects of modern computer graphics, such as visual realism and smooth camera transitions can easily induce Cybersickness in VR [8]. Four different theories discuss the etiology of Cybersickness considering Cybersickness to occur because of conflicts between real stimuli and virtual stimuli, postural instability, mismatch between real and virtual "up" direction and as a falsely triggered evolutionary survival mechanism [8]. Cybersickness can occur in the form of oculomotor, nausea, or disorientation [8].

In this study we are particularly interested in nausea and disorientation associated with virtual movement. We assume that the increased Cybersickness symptoms throughout past years [8] could be in part related to the development of real-time 3D graphics. More realistic visuals contain more detail and possibly increased spatial frequency and can thus produce more convincing visual stimulus. The contribution of this study is the comparison of graphics realism as well as visual detail reduction and their effect on Cybersickness during virtual movement in VR.

Virtual movement in VR is currently one of the biggest Cybersickness-related issues, since the optical flow caused by virtual movement (translation of position in VR triggered by some type of controller) easily produces vection, the visual illusion of movement; vection is one of the most certain prerequisites of $\mathrm{Cy}$ bersickness since it produces a significant sensory mismatch as oculo-vestibular conflict [3]. This produces symptoms similar to motion sickness $[3,8]$. The visual realism of the environment and number of orientation cues seem to affect the onset of Cybersickness during virtual movement due to increased conflicts with proprioceptive and vestibular senses [8]. Earlier studies have found that

*e-mail: firstname.lastname@ oulu.fi

†e-mail: lavalle@illinois.edu visual realism as well as fast moving detail seem to increase Cybersickness symptoms $[1,5]$. There are also many studies implicating that spatial frequency within VR navigation is a strong indicator for vection-based Cybersickness (eg. [4,9]). The visual complexity of the scene eventually contributes to the spatial frequency that has been seen as a useful generic metric for quantifying scene complexity in navigation [9].

\section{Research Method}

In this study we were interested in examining the effect of varying conditions of visual realism on Cybersickness, specifically during virtual movement. Our hypothesis was that realistic graphics would contain more realistic stimuli for virtual movement and for that reason, would induce more Cybersickness. For testing our hypothesis, we developed two VR applications containing near-identical scenes but with varying graphical styles.

\subsection{Research Conditions}

The conditions were defined as high-realism (modern graphics) and no-detail (removing small detail altogether using post-processing). Unreal Engine 4.15 was used for the development of the applications and Oculus CV1 was utilized as the VR HMD. Neither application had sound. The scene depicts a real world location of Kizhi island outdoor museum that is located in lake Onega, near Petrozavodsk, Russia. The scene contains a dynamic directional light casting realtime shadows, an ambient light as well as pre-rendered lightmaps. The scene also contains an atmospheric fog effect. An interior of a building also contains additional lights for simulating sunlight filtering through the windows. Post-processing such as motion blur and HDR lighting is utilized. The parameters of the directional light, ambient light, the fog effect as well as the dynamic skybox were controlled utilizing a commercial Unreal Blueprint collection "Ultra Dynamic Sky". Trees, foliage and terrain material were acquired from a commercial asset package "Country Side" and utilize PBR materials. Foliage and trees, as well as the lake surface is animated. A screenshot of the high-realism scene can be seen in Figure 1. In the "no-detail" condition, visual detail was reduced using the celshading technique [2]. The effect was achieved by replacing texture maps with color constants and applying a post-processing material in Unreal Engine to manipulate lighting. The post-process material was generated according to the Epic Games tutorial on cel-shading post processing. A screenshot of the no-detail scene can be seen in Figure 1.

\subsection{The Experiments}

Controlled experiments were conducted to investigate the onset of Cybersickness between the graphical conditions. In both conditions, a preprogrammed route was utilized so that all subjects would pass the same scenery and subjects of a particular condition would receive similar visual stimuli. It took a total of 15 minutes to pass through the route. While the the participants' virtual location was translated automatically, the participants were able to look around freely during immersion. The overall speed was adjusted as somewhat relaxed to mimic a walking pace in contemporary video games. The route utilized by each condition can be seen in Figure 1 .

Experimental data was collected with questionnaires. Cybersickness was quantified utilizing the Fast Motion Sickness Scale (FMS) [7], as well as the Simulator Sickness Questionnaire (SSQ) [6]. FMS 


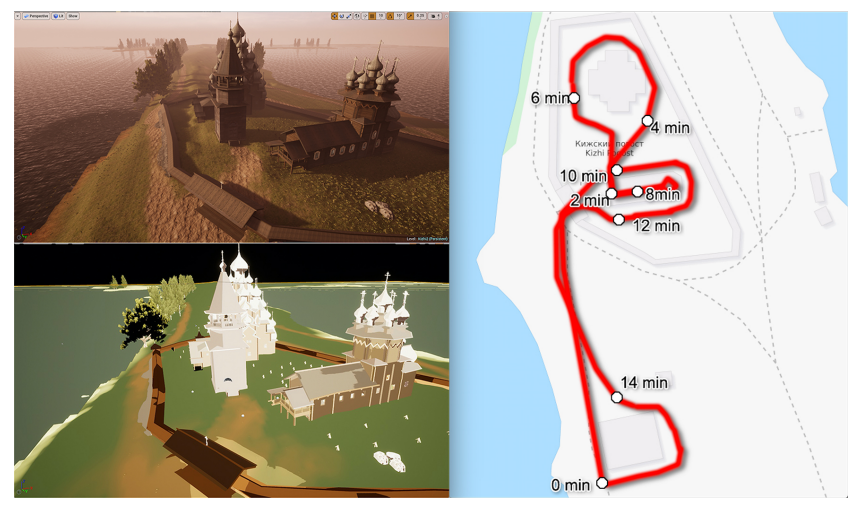

Figure 1: A scene overview for high realism (top) and no-detail (bottom). Experiment route with FMS question locations (right)

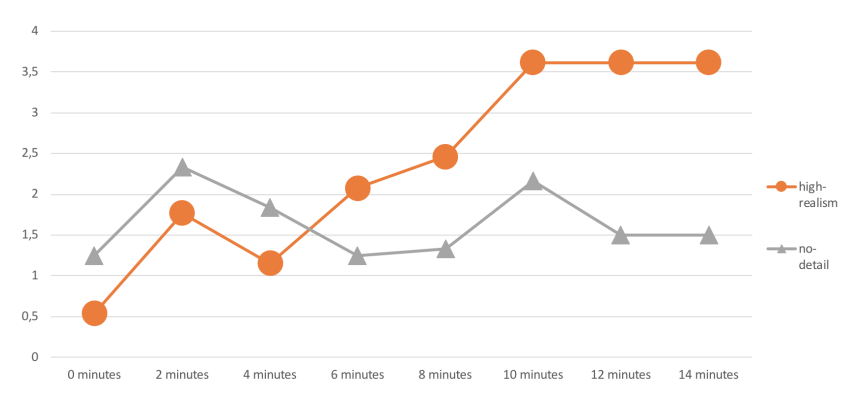

Figure 2: A timeline visualization depicting mean FMS scores.

data was collected by asking each participant to state their present illness on scale from 0 (no sickness) to 20 (frank sickness) every two minutes. The SSQ data is anecdotal since no pre-test SSQ scores were acquired. However, FMS scores have been found to correlate with SSQ-D and SSQ-T scores [7].

One participant assigned to high-realism condition felt too nauseous to continue at 12 minutes and had to stop the test. Similarly to [7], the remaining values were defined as the final score reported before the dropout (20). In high-realism condition, one participant also had to drop after 10 minutes because of a bug that prevented finishing the route. For this participant, the remaining values were defined as the last reported score (3) as well.

Qualitative data was also gathered by interviewing the participants after the experiment. No participants reported any sickness or conditions that would prevent participating in the test. The participants had the option to quit the experiment at any time.

\section{Analysis and Results}

We analyze data from 25 participants, of which 13 were assigned to the high-realism condition and 12 to the no-detail condition. The mean scores from FMS timeline (Fig. 2) and FMS peak (Table 2) imply the high-realism condition causing more symptoms. However, the data did not yield any statistical significant findings. The FMS timeline data satisfies the normality condition but the Student's t-test (two-tailed p $0.15>0.05$ ) does not cross the level of significance. The peak FMS scores did not satisfy the normality requirement, thus the Mann-Whitney test was performed with results p $0.29>0.05$ (two tailed), which also fails to cross the level of significance. Since the SSQ scores were obtained without a pre-test, their significance are not analyzed and are presented here for anecdotal evidence only (Table 1).

Inspecting the FMS timeline scores further revealed that the peak scores occurred at 2 minute and 10 minute marks. These times
Table 1: Mean SSQ scores including the SSQ subscales.

\begin{tabular}{|l|c|c|c|c|}
\hline Total & SSQ-N & SSQ-O & SSQ-D & SSQ-T \\
\hline high-realism & 29 & 26 & 41 & 358 \\
\hline no-detail & 14 & 16 & 24 & 225 \\
\hline
\end{tabular}

Table 2: Mean peak FMS scores.

\begin{tabular}{|l|c|c|c|}
\hline & Male & Female & All \\
\hline high-realism & 4.33 & 6.50 & 4.69 \\
\hline no-detail & 2.30 & 3.14 & 3.08 \\
\hline
\end{tabular}

coincide the times in which the participants were entering and exiting a building through a small set of stairs. This is in line with the qualitative data gathered; according to the interviews, the majority of the participants considered moving through the staircase the most sickness-inducing part of the experiment.

\section{Conclusion}

We studied the onset of Cybersickness in a similar scene utilizing two different graphics conditions: high-realism and no-detail. We found weak evidence from FMS timeline, FMS peak and SSQ scores for high-realism being more symptom-inducing, however, we were unable to prove statistical significance. In addition, we found that the subjects considered ascending and descending stairs and being inside a building being the most sickness-inducing parts of the experiment. We plan to investigate this topic further in the near future with more careful experiment design and larger sample size.

\section{ACKNOWLEDGMENTS}

This work was supported by the COMBAT project (293389) funded by the Strategic Research Council at the Academy of Finland.

\section{REFERENCES}

[1] N. K. Davis S. and E. Nalivaiko. Comparing the onset of cybersickness using the Oculus Rift and two virtual roller coasters. In N. K. Pisan Y. and K. Blackmore, eds., 11th Australasian Conference on Interactive Entertainment (IE 2015), vol. 167 of CRPIT, pp. 3-14. ACS, Sydney, Australia, 2015.

[2] P. Decaudin. Cartoon-looking rendering of 3D-scenes. 1996.

[3] L. J. Hettinger, K. S. Berbaum, R. S. Kennedy, W. P. Dunlap, and M. D. Nolan. Vection and simulator sickness. Military Psychology, 2(3):171-181, 1990.

[4] S. Hu, M. Davis, A. Klose, E. Zabinsky, S. Meux, H. Jacobsen, J. Westfall, and M. Gruber. Effects of spatial frequency of a vertically striped rotating drum on vection-induced motion sickness. Aviation, space, and environmental medicine, 68(4):306-311, April 1997.

[5] D. M. Johnson. Introduction to and Review of Simulator Sickness Research. Technical report, 2005.

[6] R. S. Kennedy, N. E. Lane, K. S. Berbaum, and M. G. Lilienthal. Simulator Sickness Questionnaire: An Enhanced Method for Quantifying Simulator Sickness, 1993.

[7] B. Keshavarz and H. Hecht. Validating an efficient method to quantify motion sickness. Human factors, 53(4):415-26, aug 2011.

[8] L. Rebenitsch and C. Owen. Review on cybersickness in applications and visual displays. Virtual Reality, 20(2):101-125, jun 2016.

[9] R. H. So, A. Ho, and W. Lo. A metric to quantify virtual scene movement for the study of cybersickness: Definition, implementation, and verification. Presence: Teleoperators and Virtual Environments, 10(2):193-215, 2001.

[10] K. M. Stanney and R. S. Kennedy. The psychometrics of cybersickness. Communications of the ACM, 40(8):66-68, 1997.

[11] K. M. Stanney, R. S. Kennedy, and J. M. Drexler. Cybersickness is Not Simulator Sickness. Proceedings of the Human Factors and Ergonomics Society Annual Meeting, 41(2):1138-1142, 1997. 